TITLE:

\title{
Divergence of Viscosity in Jammed Granular Materials: A Theoretical Approach
}

$\operatorname{AUTHOR}(S):$

Suzuki, Koshiro; Hayakawa, Hisao

CITATION:

Suzuki, Koshiro ... [et al]. Divergence of Viscosity in Jammed Granular Materials: A

Theoretical Approach. Physical Review Letters 2015, 115(9): 098001.

ISSUE DATE:

2015-08-28

URL:

http://hdl.handle.net/2433/232498

RIGHT:

(C) 2015 American Physical Society. 


\title{
Divergence of Viscosity in Jammed Granular Materials: A Theoretical Approach
}

\author{
Koshiro Suzuki* \\ Analysis Technology Development Center, Canon Inc., 30-2 Shimomaruko 3-chome, Ohta-ku, Tokyo 146-8501, Japan \\ Hisao Hayakawa \\ Yukawa Institute for Theoretical Physics, Kyoto University, Kitashirakawaoiwake-cho, Sakyo-ku, Kyoto 606-8502, Japan
}

(Received 8 June 2015; published 24 August 2015)

\begin{abstract}
A theory for jammed granular materials is developed with the aid of a nonequilibrium steady-state distribution function. The approximate nonequilibrium steady-state distribution function is explicitly given in the weak dissipation regime by means of the relaxation time. The theory quantitatively agrees with the results of the molecular dynamics simulation on the critical behavior of the viscosity below the jamming point without introducing any fitting parameter.
\end{abstract}

DOI: 10.1103/PhysRevLett.115.098001

Introduction.-A description of granular rheology has been a long-term challenge for both science and technology. The problem extends to a vast range, from solidlike creep motion, gaslike, to liquidlike phenomena [1]. Similar to solid-liquid transitions, granular materials acquire rigidity when the density exceeds a critical value [2-5]. This phenomenon, referred to as the jamming transition, is universely observed in disordered materials such as colloidal suspensions [6], emulsions, and foams [7], as well as granular materials. The jamming transition and its relation to the glass transition have attracted much interest in the last two decades, and various aspects have been revealed [8-12]. In particular, characteristics in the vicinity of the jamming point, including the critical scaling behavior, have been extensively investigated by experiments and numerical simulations [2-4,13-25]. It has been shown that the shear stress, the pressure, and the granular temperature can be expressed by scaling functions with exponents near $\varphi \sim \varphi_{J}$, where $\varphi_{J}$ is the jamming transition density. The shear viscosity exhibits a form $\eta \sim\left(\varphi_{J}-\varphi\right)^{-\lambda}$ with $\lambda \approx 2$ for non-Browninan suspensions, foams, and emulsions [26-29], and a recent careful analysis demonstrated that $\lambda$ is in the range between 1.67 and 2.55 [30]. It seems that the exponent $\lambda$ for granular flows takes a larger value than that for suspensions $[18,19,25,31]$, although a value in the range mentioned above has been reported as well [17]. However, these studies are based on numerical simulations or phenomenologies without any foundation of a microscopic theory.

Even when we focus only on the flow properties below the jamming point $\varphi_{J}$, which can be tracked back to Bagnold's work [32], we have not yet obtained a complete set in describing the rheological properties of dense granular flows. One of the remarkable achievements is the extension of the Boltzmann-Enskog kinetic theory to inelastic hard disks and spheres [33-38]. However, it has been recognized that the kinetic theory breaks down at densities with volume fraction $\varphi>\varphi_{f}=0.49$ [39-42],
PACS numbers: 45.70.-n, 05.20.Jj, 64.70.ps, 83.50.Ax

since there exists correlated motions of grains. A modification of the kinetic theory has been proposed [43], but a microscopic theory is still absent.

Because of these situations, a microscopic liquid theory valid in the regime $\varphi_{f}<\varphi<\varphi_{\mathrm{J}}$ has been desired. One attempt to respond to this problem is the extension of the mode-coupling theory (MCT) [44] for dense granular liquids. MCT has been applied to granular systems driven by Gaussian noises [45-47]. It qualitatively predicts a liquid-glass transition, though the noise in granular systems is non-Gaussian in general [48-51]. MCT also has been applied to sheared dense granular systems [52-54]. There are three disadvantages of this approach: (i) the shift of $\varphi$ is necessary to describe the divergence of $\eta$. (ii) Because of the shift of $\varphi$, the jamming transition is not correlated with the divergence of the first peak of the radial distribution function. (iii) It predicts a plateau in the density correlation function, which is not observed in experiments nor in simulations [55-57].

From these observations, it is crucial to obtain an explicit expression of the steady-state distribution function to construct a theory for dense granular liquids. For our purpose, we attempt to perform an expansion with respect to the dissipation to obtain an approximate explicit expression of the distribution function, valid in the weak dissipation regime for frictionless granular flows. Once the distribution is obtained explicitly, it is possible to calculate the steady-state average for arbitrary observables.

Microscopic starting equations.-We consider a threedimensional system of $N$ smooth granular particles of mass $m$ and diameter $d$ in a volume $V$ subjected to stationary shearing characterized by the shear-rate tensor $\dot{\gamma}$. We assume that each granular particle is a soft-sphere, and the contact force acts only on the normal direction. For a simple uniform shear with velocity along the $x$ axis and its gradient along the $y$ axis, the shear-rate tensor is $\dot{\gamma}_{\mu \nu}=\dot{\gamma} \delta_{\mu x} \delta_{\nu y}(\mu, \nu=x, y, z)$ with a shear rate $\dot{\gamma}$. It is postulated that the applied shear induces a homogeneous streaming-velocity profile $\dot{\gamma} \cdot \boldsymbol{r}$ at 
position $\boldsymbol{r}$, assuming that no heterogeneity such as shear banding exists. Thus, the equation of motion is given by

$$
\begin{aligned}
& \boldsymbol{p}_{i}=m\left(\dot{\boldsymbol{r}}_{i}-\dot{\boldsymbol{\gamma}} \cdot \boldsymbol{r}_{i}\right), \\
& \dot{\boldsymbol{p}}_{i}=\boldsymbol{F}_{i}^{(\mathrm{el})}+\boldsymbol{F}_{i}^{(\mathrm{vis})}-\dot{\gamma} \cdot \boldsymbol{p}_{i},
\end{aligned}
$$

where $\boldsymbol{F}_{i}^{(\mathrm{el})}$ and $\boldsymbol{F}_{i}^{(\mathrm{vis})}$ are, respectively, the elastic and the viscous contact forces acting on the grain $i$. Equation (1) is known as the Sllod equation, which is equivalent to Newton's equation of motion under a uniform shear [58].

The most essential feature of granular systems, in contrast to thermal systems, is that the steady state is determined by the balance between the viscous heating and the energy dissipation due to inelastic collisions. For sheared granular systems, this can be seen from the time derivative of the Hamiltonian, $\mathcal{H}(\boldsymbol{\Gamma})=\sum_{i=1}^{N} \boldsymbol{p}_{i}^{2} /(2 m)+$ $\sum_{i, j}{ }^{\prime} u\left(r_{i j}\right)$, where $u\left(r_{i j}\right)$ is the interparticle potential depending on $r_{i j}=\left|\boldsymbol{r}_{i}-\boldsymbol{r}_{j}\right|, \quad \boldsymbol{\Gamma}=\left\{\boldsymbol{r}_{i}, \boldsymbol{p}_{i}\right\}_{i=1}^{N}$ is the phase-space coordinate, and $\sum_{i, j}{ }^{\prime}$ is the summation under the condition $i \neq j$. Then $\dot{\mathcal{H}}(\boldsymbol{\Gamma})$ satisfies

$$
\dot{\mathcal{H}}(\boldsymbol{\Gamma})=-\dot{\gamma} V \sigma_{x y}(\boldsymbol{\Gamma})-2 \mathcal{R}(\boldsymbol{\Gamma}),
$$

where

$$
\sigma_{\mu \nu}(\boldsymbol{\Gamma})=\frac{1}{V} \sum_{i=1}^{N}\left[\frac{p_{i, \mu} p_{i, \nu}}{m}+r_{i, \nu}\left(F_{i, \mu}^{(\mathrm{el})}+F_{i, \mu}^{(\mathrm{vis})}\right)\right]
$$

is the stress tensor and

$$
\mathcal{R}(\boldsymbol{\Gamma})=-\frac{1}{2} \sum_{i=1}^{N} \dot{\boldsymbol{r}}_{i} \cdot \boldsymbol{F}_{i}^{(\mathrm{vis})}
$$

corresponds to the Rayleigh's dissipation function $[59,60]$. For granular systems with the interparticle dissipative force proportional to the relative velocity, it is impossible to reduce the dynamics as overdamped. For later analysis, we assume that the contact forces $\boldsymbol{F}_{i}^{(\mathrm{el})}=\sum_{j \neq i} \boldsymbol{F}_{i j}^{(\mathrm{el})}$ and $\boldsymbol{F}_{i}^{(\mathrm{vis})}=$ $\sum_{j \neq i} \boldsymbol{F}_{i j}^{(\mathrm{vis})}$ are, respectively, given by $\boldsymbol{F}_{i j}^{(\mathrm{el})}=\kappa \Theta\left(d-r_{i j}\right) \times$ $\left(d-r_{i j}\right)$ and $\boldsymbol{F}_{i j}^{(\mathrm{vis})}=-\zeta \Theta\left(d-r_{i j}\right)\left(\boldsymbol{v}_{i j} \cdot \hat{\boldsymbol{r}}_{i j}\right) \hat{\boldsymbol{r}}_{i j}$, where $\Theta(x)=1$ for $x \geq 0$ and $\Theta(x)=0$ otherwise, $\boldsymbol{r}_{i j}=\boldsymbol{r}_{i}-\boldsymbol{r}_{j}, \hat{\boldsymbol{r}}_{i j}=\boldsymbol{r}_{i j} / r_{i j}$, and $\boldsymbol{v}_{i j}=\boldsymbol{v}_{i}-\boldsymbol{v}_{j}$ with the velocity of $i$ th particle $\boldsymbol{v}_{i}$.

Steady-state distribution function.-To address the distribution function for the nonequilibrium steady state, we start from an equilibrium state at $t \rightarrow-\infty$ and evolve the system with shear and dissipation. Then, the system is expected to reach a steady state at $t=0$. Although it is impossible to derive an exact solution of the Liouville equation, equivalent to Eq. (1), for the $6 N$-dimensional distribution function, it is possible to obtain an approximate solution by perturbation, parallel to the method for the linearized Boltzmann equation [70]. In the perturbation for dense sheared granular systems, it is simple to obtain the leading-order eigenfrequency of the relaxation towards the steady state [60]. Hence, we attempt to speculate an approximate steady-state distribution, which we denote
$\rho_{\mathrm{SS}}(\boldsymbol{\Gamma})$, by applying an approximation which explicitly utilizes the relaxation time.

For this purpose, we start from a formal but exact expression for the distribution function [58],

$$
\rho_{\mathrm{SS}}^{(\mathrm{ex})}(\boldsymbol{\Gamma})=\exp \left[\int_{-\infty}^{0} d \tau \boldsymbol{\Omega}_{\mathrm{eq}}[\boldsymbol{\Gamma}(-\tau)]\right] \rho_{\mathrm{eq}}[\boldsymbol{\Gamma}(-\infty)],
$$

which is the steady-state solution of the Liouville equation. Here, $\Omega_{\text {eq }}(\boldsymbol{\Gamma})=\beta_{\text {eq }} \dot{\mathcal{H}}(\boldsymbol{\Gamma})-\Lambda(\boldsymbol{\Gamma})=-\beta_{\text {eq }}\left[\dot{\gamma} V \sigma_{x y}(\boldsymbol{\Gamma})+2 \mathcal{R}(\boldsymbol{\Gamma})\right]-$ $\Lambda(\boldsymbol{\Gamma})$ is the work function for $\rho_{\mathrm{eq}}(\boldsymbol{\Gamma})=e^{-\beta_{\mathrm{eq}} \mathcal{H}(\boldsymbol{\Gamma})} /$ $\int d \boldsymbol{\Gamma} e^{-\beta_{\text {eq }} \mathcal{H}(\boldsymbol{\Gamma})}$ at temperature $T_{\text {eq }}=\beta_{\text {eq }}^{-1}$, where $\Lambda(\boldsymbol{\Gamma})=(\partial / \partial \boldsymbol{\Gamma})$. $\dot{\boldsymbol{\Gamma}}$ is the phase-space volume contraction. We approximate Eq. (5) by introducing the relaxation time $\tau_{\text {rel }}$ as

$$
\exp \left[\int_{-\infty}^{0} d \tau \Omega_{\mathrm{eq}}[\boldsymbol{\Gamma}(-\tau)]\right] \approx e^{\tau_{\mathrm{rel}} \Omega_{\mathrm{SS}}(\boldsymbol{\Gamma})}
$$

which can be validated in the perturbation expansion of the Liouville equation around the canonical distribution [60]. In the perturbation, we nondimensionalize all the quantities, where the units of mass, length, and time are chosen as $m, d$, and $\sqrt{m / \kappa}$, and introduce $\epsilon \equiv \zeta / \sqrt{\kappa m} \ll 1$ as a perturbation parameter, which is related to the restitution coefficient $e$ as $\epsilon \approx \sqrt{2}(1-e) / \pi$ for $e \approx 1$. We attach a star $*$ to the nondimensionalized quantites, e.g., $t^{*}=t \sqrt{\kappa / m}$. Furthermore, we perform a scaling which leaves the steady-state temperature $T_{\mathrm{SS}}$, which is the ensemble average of $\sum_{i=1}^{N} \boldsymbol{p}_{i}^{2} /(3 \mathrm{Nm})$ at the steady state in the dimensional unit, to be independent of $\epsilon$. This indicates that the granular fluid keeps its motion in the limit $\epsilon \rightarrow 0$. From dimensional analysis, $T_{\mathrm{SS}}$ satisfies $T_{\mathrm{SS}} \sim m^{3} d^{2} \dot{\gamma}^{4} / \zeta^{2}$, which leads to $T_{\mathrm{SS}}^{*} \sim \epsilon^{-2} \dot{\gamma}^{* 4}$. Hence, $\dot{\gamma}^{*}$ should satisfy $\dot{\gamma}^{*} \sim \epsilon^{1 / 2}$. We introduce a scaled shear rate $\tilde{\dot{\gamma}}$ as $\dot{\gamma}^{*}=\epsilon^{1 / 2} \tilde{\dot{\gamma}}$, where $\tilde{\dot{\gamma}}$ is independent of $\epsilon$. We attach a tilde to the scaled quantities. The relaxation time $\tau_{\text {rel }}$ is evaluated from the eigenfrequency of the perturbation expansion as

$$
\tau_{\text {rel }}=\left[\frac{2 \sqrt{\pi}}{3} \epsilon \omega_{E}\left(T_{\mathrm{SS}}\right)\right]^{-1}
$$

in the hard-core limit [60], where $\omega_{E}(T)=$ $4 \sqrt{\pi} n \sqrt{T / m} g_{0}(\varphi) d^{2}$ is the Enskog frequency of collisions and $g_{0}(\varphi)$ is the first-peak value of the radial distribution function. In Eq. (6), we have also introduced

$$
\Omega_{\mathrm{SS}}(\boldsymbol{\Gamma})=-\beta_{\mathrm{SS}}\left[\dot{\gamma} V \sigma_{x y}^{(\mathrm{el})}(\boldsymbol{\Gamma})+2 \Delta \mathcal{R}_{\mathrm{SS}}^{(1)}(\boldsymbol{\Gamma})\right],
$$

where $\sigma_{x y}^{(\mathrm{el})}$ and $\Delta R_{\mathrm{SS}}^{(1)}$ are, respectively, given by [60]

$$
\begin{aligned}
\sigma_{x y}^{(\mathrm{el})}(\boldsymbol{\Gamma}) & =\frac{1}{V} \sum_{i=1}^{N}\left[\frac{p_{i, x} p_{i, y}}{m}+y_{i} F_{i, x}^{(\mathrm{el})}\right], \\
\Delta \mathcal{R}_{\mathrm{SS}}^{(1)}(\boldsymbol{\Gamma}) & =\mathcal{R}^{(1)}(\boldsymbol{\Gamma})+\frac{T_{\mathrm{SS}}}{2} \Lambda(\boldsymbol{\Gamma}), \\
\mathcal{R}^{(1)}(\boldsymbol{\Gamma}) & =\frac{\zeta}{4} \sum_{i, j}^{\prime}\left(\frac{\boldsymbol{p}_{i j}}{m} \cdot \hat{\boldsymbol{r}}_{i j}\right)^{2} \Theta\left(d-r_{i j}\right) .
\end{aligned}
$$


Here we ignore the contribution from the viscous shear stress, which is a higher-order correction in the limit $\epsilon \rightarrow 0$. To summarize, we obtain

$$
\rho_{\mathrm{SS}}(\boldsymbol{\Gamma})=\frac{e^{-I_{\mathrm{SS}}(\boldsymbol{\Gamma})}}{\int d \boldsymbol{\Gamma} e^{-I_{\mathrm{SS}}(\boldsymbol{\Gamma})},}
$$

where $I_{\mathrm{SS}}(\boldsymbol{\Gamma})=\beta_{\mathrm{SS}}^{*} \mathcal{H}^{*}(\boldsymbol{\Gamma})-\tilde{\tau}_{\text {rel }} \tilde{\Omega}_{\mathrm{SS}}(\boldsymbol{\Gamma})$ with $\tilde{\Omega}_{\mathrm{SS}}(\boldsymbol{\Gamma})=$ $-\beta_{\mathrm{SS}}^{*}\left[\tilde{\dot{\gamma}} V^{*} \tilde{\sigma}_{x y}^{(\mathrm{el})}(\boldsymbol{\Gamma})+2 \Delta \tilde{\mathcal{R}}_{\mathrm{SS}}^{(1)}(\boldsymbol{\Gamma})\right]$. We note that (i) the steady-state temperature $T_{\mathrm{SS}}=\beta_{\mathrm{SS}}^{-1}$ appears in Eq. (12), (ii) the steady-state average $\langle\cdots\rangle_{\mathrm{SS}} \equiv \int d \boldsymbol{\Gamma} \rho_{\mathrm{SS}}(\boldsymbol{\Gamma}) \cdots$ is independent of the equilibrium temperature for $\rho_{\mathrm{eq}}(\boldsymbol{\Gamma})$, (iii) the problem reduces to an "equilibrium" one with an effective Hamiltonian $\mathcal{H}_{\text {eff }}(\boldsymbol{\Gamma})=T_{\mathrm{SS}} I_{\mathrm{SS}}(\boldsymbol{\Gamma})$ and the temperature $T_{\mathrm{SS}}$. Because the nonequilibrium contribution is small, we further expand $\rho_{\mathrm{SS}}(\boldsymbol{\Gamma})$ as

$$
\rho_{\mathrm{SS}}(\boldsymbol{\Gamma}) \approx \frac{e^{-\beta_{\mathrm{SS}}^{*} \mathcal{H}^{*}(\boldsymbol{\Gamma})}\left[1+\tilde{\tau}_{\mathrm{rel}} \tilde{\Omega}_{\mathrm{SS}}(\boldsymbol{\Gamma})\right]}{\mathcal{Z}}
$$

with $\mathcal{Z} \approx \int d \boldsymbol{\Gamma} e^{-\beta_{\mathrm{SS}}^{*} \mathcal{H}^{*}(\boldsymbol{\Gamma})}\left[1+\tilde{\tau}_{\text {rel }} \tilde{\Omega}_{\mathrm{SS}}(\boldsymbol{\Gamma})\right]$. An approximate expression for $\langle A(\boldsymbol{\Gamma})\rangle_{\mathrm{SS}}$ is obtained as

$$
\langle A(\boldsymbol{\Gamma})\rangle_{\mathrm{SS}} \approx\langle A(\boldsymbol{\Gamma})\rangle_{\mathrm{eq}}+\tilde{\tau}_{\text {rel }}\left\langle A(\boldsymbol{\Gamma}) \tilde{\Omega}_{\mathrm{SS}}(\boldsymbol{\Gamma})\right\rangle_{\mathrm{eq}},
$$

where $\langle\cdots\rangle_{\mathrm{eq}}=\int d \boldsymbol{\Gamma} e^{-\beta_{\mathrm{sS}}^{*} \mathcal{H}^{*}(\boldsymbol{\Gamma})} \cdots$ is the average with respect to the canonical distribution at $T_{\mathrm{SS}}$. It should be noted that Eq. (14) is the result of an exponential damping in the stressstress correlation function in the Green-Kubo formula.

So far $T_{\mathrm{SS}}$ is undetermined. We attempt to determine $T_{\mathrm{SS}}$ by imposing the energy balance

$$
\langle\dot{\mathcal{H}}(\boldsymbol{\Gamma})\rangle_{\mathrm{SS}}=-\dot{\gamma} V\left\langle\sigma_{x y}(\boldsymbol{\Gamma})\right\rangle_{\mathrm{SS}}-2\langle\mathcal{R}(\boldsymbol{\Gamma})\rangle_{\mathrm{SS}}=0 .
$$

The explicit form of $T_{\mathrm{SS}}$ will be given in Eq. (16).

Shear viscosity and temperature.-Now we calculate the steady-state average of the shear stress and the energy dissipation rate by Eq. (14) and derive an explicit expression for $T_{\mathrm{SS}}$. First, $\left\langle\sigma_{x y}(\boldsymbol{\Gamma})\right\rangle_{\mathrm{SS}}$ is approximately given by $\left\langle\tilde{\sigma}_{x y}(\boldsymbol{\Gamma})\right\rangle_{\text {SS }} \approx-\tilde{\tau}_{\text {rel }} \tilde{\dot{\gamma}} \beta_{\text {SS }}^{*} V^{*}\left\langle\tilde{\boldsymbol{\sigma}}_{x y}^{(\mathrm{el})}(\boldsymbol{\Gamma}) \tilde{\sigma}_{x y}^{(\mathrm{el})}(\boldsymbol{\Gamma})\right\rangle_{\mathrm{eq}}$. Similarly, the leading contribution gives $\langle\tilde{\mathcal{R}}(\boldsymbol{\Gamma})\rangle_{\mathrm{SS}} \approx\left\langle\tilde{\mathcal{R}}^{(1)}(\boldsymbol{\Gamma})\right\rangle_{\mathrm{eq}}-$ $2 \tilde{\tau}_{\text {rel }} \beta_{\text {SS }}^{*}\left\langle\tilde{\mathcal{R}}^{(1)}(\boldsymbol{\Gamma}) \Delta \tilde{\mathcal{R}}_{\text {SS }}^{(1)}(\boldsymbol{\Gamma})\right\rangle_{\text {eq }}$. Thus, we obtain the steadystate temperature from Eq. (15) as

$$
T_{\mathrm{SS}}^{*}=\frac{3 \tilde{\dot{\gamma}}^{2}}{32 \pi} \frac{S}{R},
$$

where $S$ and $R$ are given by $S=1+\mathcal{S}_{2} n^{*} g_{0}(\varphi)+$ $\mathcal{S}_{3} n^{* 2} g_{0}(\varphi)^{2}+\mathcal{S}_{4} n^{* 3} g_{0}(\varphi)^{3} \quad$ and $\quad R=n^{*} g_{0}(\varphi)\left[\mathcal{R}_{2}^{\prime}+\right.$ $\left.\mathcal{R}_{3}^{\prime} n^{*} g_{0}(\varphi)\right]$, with $\mathcal{S}_{2}=2 \pi / 15, \mathcal{S}_{3}=-\pi^{2} / 20, \mathcal{S}_{4}=3 \pi^{3} / 160$, $\mathcal{R}_{2}^{\prime}=-3 / 4$, and $\mathcal{R}_{3}^{\prime}=7 \pi / 16$ [60]. We adopt the interpolation formula for hard spheres valid in the range $\varphi_{f}<\varphi<\varphi_{J} \quad\left(\varphi_{f}=0.49, \quad \varphi_{J}=0.639\right), g_{0}(\varphi)=$ $g_{\mathrm{CS}}\left(\varphi_{f}\right)\left(\varphi_{f}-\varphi_{J}\right) /\left(\varphi-\varphi_{J}\right)$, where $g_{\mathrm{CS}}(\varphi)=(1-\varphi / 2) /$ $(1-\varphi)^{3}$ is the formula by Carnahan and Starling valid at $\varphi<\varphi_{f}$ [71]. Note that we adopt the Kirkwood approximation for many-body correlations [60]. We further obtain the expression for the shear stress,

$$
\left\langle\tilde{\sigma}_{x y}(\boldsymbol{\Gamma})\right\rangle_{\mathrm{SS}}=-\frac{3 \sqrt{6}}{64 \pi^{3 / 2}} \tilde{\dot{\gamma}}^{2} \frac{S^{3 / 2}}{R^{1 / 2} g_{0}(\varphi)} .
$$

In the vicinity of the jamming point $\varphi_{J}, S$ and $R$ can be approximated as $S \approx \mathcal{S}_{4} n^{* 3} g_{0}(\varphi)^{3}$ and $R \approx \mathcal{R}_{3}^{\prime} n^{* 2} g_{0}(\varphi)^{2}$, respectively. This leads to the following expressions,

$$
\begin{aligned}
T_{\mathrm{SS}}^{*} & \approx \frac{9 \pi}{2240} \tilde{\dot{\gamma}}^{2} n^{*} g_{0}(\varphi), \\
\left\langle\tilde{\sigma}_{x y}(\boldsymbol{\Gamma})\right\rangle_{\mathrm{SS}} & \approx-\frac{27 \pi^{5 / 2}}{10240 \sqrt{35}} \tilde{\dot{\gamma}}^{2} n^{* 7 / 2} g_{0}(\varphi)^{5 / 2},
\end{aligned}
$$

from which we obtain

$$
\begin{aligned}
T_{\mathrm{SS}}^{*} & \sim\left(\varphi_{J}-\varphi\right)^{-1}, \\
\left\langle\tilde{\sigma}_{x y}(\boldsymbol{\Gamma})\right\rangle_{\mathrm{SS}} & \sim\left(\varphi_{J}-\varphi\right)^{-5 / 2} .
\end{aligned}
$$

From Eq. (17), we obtain the shear viscosity $\eta^{*}=$ $-\left\langle\tilde{\sigma}_{x y}(\boldsymbol{\Gamma})\right\rangle_{\mathrm{SS}} / \tilde{\dot{\gamma}} \sim\left(\varphi_{J}-\varphi\right)^{-5 / 2}$, or for $\tilde{\eta}^{\prime}=-\left\langle\tilde{\sigma}_{x y}(\boldsymbol{\Gamma})\right\rangle_{\mathrm{SS}} /$ $\left(\tilde{\dot{\gamma}} \sqrt{T_{\mathrm{SS}}^{*}}\right) \propto-\left\langle\tilde{\sigma}_{x y}(\boldsymbol{\Gamma})\right\rangle_{\mathrm{SS}} / \tilde{\dot{\gamma}}^{2}$,

$$
\tilde{\eta}^{\prime} \sim\left(\varphi_{J}-\varphi\right)^{-2} .
$$

These results in Eqs. (20)-(22) are consistent with the previous observations [26-30].

Comparison with simulation.-In order to verify the validity of the theory, we compare the theory with the molecular dynamics (MD) simulation. The parameters in the MD are $N=2000, \epsilon=0.018375$, and $\dot{\gamma}^{*}=10^{-3}, 10^{-4}$, $10^{-5}$. This condition corresponds to $e=0.96$.

The shear viscosity $\tilde{\eta}^{\prime}$ and $T_{\mathrm{SS}}$ are shown in Fig. 1, together with the results of the MD. We also show the

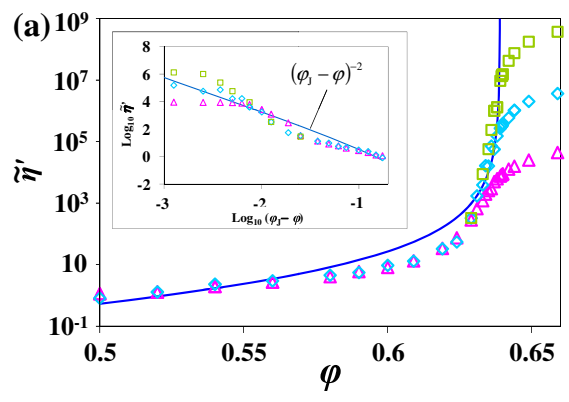

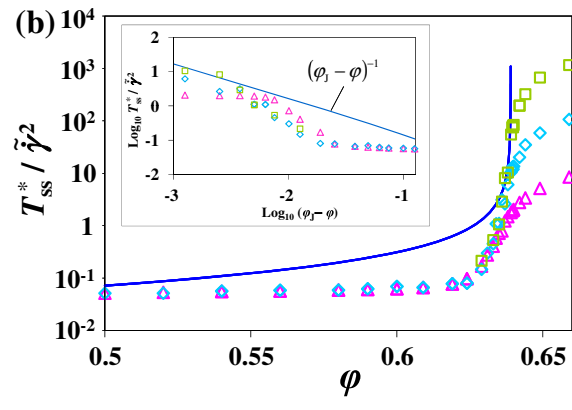

FIG. 1 (color online). The density dependence of (a) the shear viscosity $\tilde{\eta}^{\prime}$ and (b) the granular temperature. The result of the theory is shown in the (blue) solid line, while that for the MD is shown in (red) triangles, (blue) diamonds, and (green) rectangles for $\dot{\gamma}^{*}=10^{-3}, 10^{-4}, 10^{-5}$. (Inset) The log-log plots for the results near $\varphi_{J}=0.639$. 


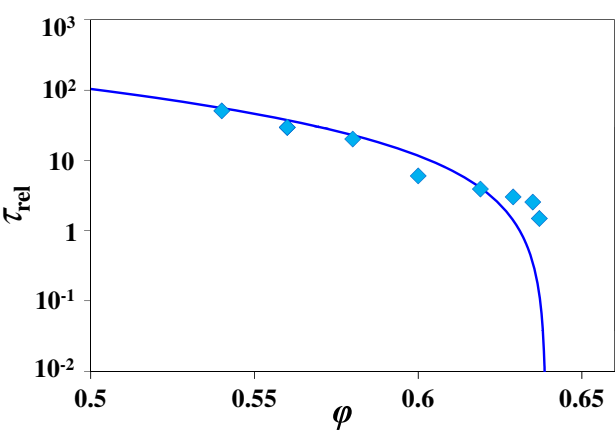

FIG. 2 (color online). The density dependence of the relaxation time, $\tilde{\tau}_{\text {rel }}=\epsilon \tau_{\text {rel }}^{*}$. The result for the shear rate $\dot{\gamma}^{*}=10^{-4}$ is shown in the (blue) solid line for the theory and (blue) diamonds for the MD simulation.

$\log -\log$ plots near $\varphi_{J}$ as a function of $\varphi_{J}-\varphi$ in the inset. From the figure, we see that the theory agrees with the result of MD simulation for $\varphi<\varphi_{J}$ quantitatively, without introducing any fitting parameter. The agreement is refined as the shear rate is decreased, where the hard-core limit is realized asymptotically. The smeared divergence in the vicinity of the jamming point observed for finite $\dot{\gamma}^{*}$ is a well-known feature of the soft-core MD. We stress that the theory predicts the divergence of both the shear viscosity and the granular temperature as $\varphi \rightarrow \varphi_{J}$, in contrast to the kinetic theory of inelastic spheres, where the shear viscosity behaves as $\tilde{\eta}^{\prime} \sim\left(\varphi_{J}-\varphi\right)^{-1}$ and $T_{\mathrm{SS}}$ remains finite [35]. On the other hand, in the MD, the shear viscosity behaves as $\tilde{\eta}^{\prime} \sim\left(\varphi_{J}-\varphi\right)^{-2}$, in accordance with the theory. We note that the agreement between the theory and MD is relatively poor for $T_{\mathrm{SS}}$, though we have not clarified its reason.

The relaxation time, Eq. (7), is shown in Fig. 2, together with the result of the MD. The result of the MD is extracted from fitting by an exponential function for the transient data of the temperature relaxing to the steady state. We see that Eq. (7) is quantitatively valid for $\varphi<0.63$.

Discussions.-From Eqs. (16) and (17), we see that the theory is subjected to the Bagnold scaling. The result of MD shows that the discrepancy from the Bagnold scaling becomes significant for $\varphi>0.635$. Hence, there is room for improving the theory to cover the non-Bagnold regime. From the phenomenological scaling of jammed granules, the viscosity exhibits $\eta \sim\left|\varphi_{J}-\varphi\right|^{y_{\phi}\left(1-2 / y_{\gamma}\right)}$, where $y_{\phi}$ and $y_{\gamma}$ are the scaling exponents for $\sigma_{x y} \sim\left(\varphi-\varphi_{J}\right)^{y_{\phi}}$ for $\varphi>$ $\varphi_{J}$ and $\sigma_{x y} \sim \dot{\gamma}^{y_{\gamma}}$ at $\varphi=\varphi_{J}$ [18]. If we assume $y_{\phi}=1$ as in Refs. $[2,3,18,19]$, we have $y_{\gamma}=4 / 7$, which is close to the value of Refs. [17,72]. For strongly dissipative situations, higher-order terms might alter the exponents of the divergences. Such a contribution will be discussed elsewhere.

Concluding remarks. -We have developed a theory for jammed frictionless granular particles subjected to a uniform shear with the aid of an approximate nonequilibrium steady-state distribution function, and have shown that it remarkably agrees with the result of the MD simulation below the jamming point without introducing any fitting parameter. There are many future tasks for the application of our theory, such as the emergence of the shear modulus above the jamming point [2-5], the effect of friction for grains where the discontinuous shear thickening appears [73], the drag force acting on the pulling tracer [74-78], etc. Moreover, we should stress that the framework of our theory is quite generic. Indeed, we believe that the divergence of the viscosity for colloidal suspensions, $\eta \sim$ $\left(\varphi_{J}-\varphi\right)^{-2}$ [79], can be understood by our framework. Therefore, the theory is expected to be applicable to a wide variety of phenomena in nonequilibrium processes.

The authors are grateful to S.-H. Chong and M. Otsuki for their contributions in the early stage of this work and extensive discussions. We also thank M. Fuchs, M.E. Cates, M. Wyart, K. Saitoh, A. Ikeda, and K. Kanazawa for fruitful discussions and comments, and M. Otsuki and S. Takada for providing the prototype of the program for the MD simulation. This work was partially supported by Grant-in-Aids for Scientific Research (Grant No. 25287098). The MD simulation for this work has been carried out at the computer facilities at the Yukawa Institute for Theoretical Physics, Kyoto University.

*Corresponding author. koshiro@yukawa.kyoto-u.ac.jp, suzuki.koshiro@canon.co.jp

[1] H. M. Jaeger, S. R. Nagel, and R. P. Behringer, Rev. Mod. Phys. 68, 1259 (1996).

[2] C. S. O’Hern, S. A. Langer, A. J. Liu, and S. R. Nagel, Phys. Rev. Lett. 88, 075507 (2002).

[3] C. S. O’Hern, L. E. Silbert, A. J. Liu, and S. R. Nagel, Phys. Rev. E 68, 011306 (2003).

[4] M. Otsuki and H. Hayakawa, Phys. Rev. E 90, 042202 (2014).

[5] C. Coulais, A. Seguin, and O. Dauchot, Phys. Rev. Lett. 113, 198001 (2014).

[6] P. N. Pusey, in Liquids, Freezing and Glass Transition, Part II, Proceedings of the Les Houches Summer School, Vol. 51, edited by J.-P. Hansen, D. Levesque, and J. Zinn-Justin (Elsevier, Amsterdam, 1991).

[7] D. J. Durian and D. A. Weitz, in "Foams" in Kirk-Othmer Encyclopedia of Chemical Technology, edited by J.I. Kroschwitz (Wiley, New York, 1994), p. 783.

[8] A. J. Liu and S. R. Nagel, Nature (London) 396, 21 (1998).

[9] A. Ikeda, L. Berthier, and P. Sollich, Phys. Rev. Lett. 109, 018301 (2012).

[10] S. Torquato and F. H. Stillinger, Rev. Mod. Phys. 82, 2633 (2010).

[11] G. Parisi and F. Zamponi, Rev. Mod. Phys. 82, 789 (2010).

[12] A. Liu and S. R. Nagel, Annu. Rev. Condens. Matter Phys. 1, 347 (2010).

[13] F. da Cruz, S. Emam, M. Prochnow, J.-N. Roux, and F. Chevoir, Phys. Rev. E 72, 021309 (2005).

[14] M. Wyart, L. E. Silbert, S. R. Nagel, and T. A. Witten, Phys. Rev. E 72, 051306 (2005).

[15] P. Olsson and S. Teitel, Phys. Rev. Lett. 99, 178001 (2007).

[16] T. Hatano, Phys. Rev. E 75, 060301(R) (2007).

[17] T. Hatano, J. Phys. Soc. Jpn. 77, 123002 (2008). 
[18] M. Otsuki and H. Hayakawa, Prog. Theor. Phys. 121, 647 (2009).

[19] M. Otsuki and H. Hayakawa, Phys. Rev. E 80, 011308 (2009).

[20] M. Otsuki and H. Hayakawa, Phys. Rev. E 83, 051301 (2011).

[21] B. P. Tighe, E. Woldhuis, J. J. C. Remmers, W. van Saarloos, and M. van Hecke, Phys. Rev. Lett. 105, 088303 (2010).

[22] D. Bi, J. Zhang, B. Chakraborty, and R. P. Behringer, Nature (London) 480, 355 (2011).

[23] P. Olsson and S. Teitel, Phys. Rev. Lett. 109, 108001 (2012).

[24] P. Olsson and S. Teitel, Phys. Rev. E 88, 010301(R) (2013).

[25] E. DeGiuli, G. Düring, E. Lerner, and M. Wyart, Phys. Rev. E 91, 062206 (2015).

[26] F. Boyer, E. Guazzelli, and O. Pouliquen, Phys. Rev. Lett. 107, 188301 (2011).

[27] M. Trulsson, B. Andreotti, and P. Claudin, Phys. Rev. Lett. 109, 118305 (2012).

[28] B. Andreotti, J.-L. Barrat, and C. Heussinger, Phys. Rev. Lett. 109, 105901 (2012).

[29] E. Lerner, G. Düring, and M. Wyart, Proc. Natl. Acad. Sci. U.S.A. 109, 4798 (2012).

[30] T. Kawasaki, D. Coslovich, A. Ikeda, and L. Berthier, Phys. Rev. E 91, 012203 (2015).

[31] M. Otsuki, H. Hayakawa, and S. Luding, Prog. Theor. Phys. Suppl. 184, 110 (2010).

[32] R. A. Bagnold, Proc. R. Soc. A 225, 49 (1954).

[33] J. T. Jenkins and M. W. Richman, Phys. Fluids 28, 3485 (1985).

[34] J. T. Jenkins and M. W. Richman, Arch. Ration. Mech. Anal. 87, 355 (1985).

[35] V. Garzó and J. W. Dufty, Phys. Rev. E 59, 5895 (1999).

[36] J. F. Lutsko, Phys. Rev. E 72, 021306 (2005).

[37] K. Saitoh and H. Hayakawa, Phys. Rev. E 75, 021302 (2007).

[38] N. V. Brilliantov and T. Pöschel, Kinetic Theory of Granular Gases (Oxford, New York, 2010).

[39] M. Y. Louge, Phys. Rev. E 67, 061303 (2003).

[40] N. Mitarai and H. Nakanishi, Phys. Rev. Lett. 94, 128001 (2005).

[41] N. Mitarai and H. Nakanishi, Phys. Rev. E 75, 031305 (2007).

[42] J. T. Jenkins and D. Berzi, Granular Matter 12, 151 (2010).

[43] S. Chialvo and S. Sundaresan, Phys. Fluids 25, 070603 (2013).

[44] W. Götze, Complex Dynamics of Glass-Forming Liquids: A Mode-Coupling Theory (Oxford, New York, 2009).

[45] W. T. Kranz, M. Sperl, and A. Zippelius, Phys. Rev. Lett. 104, 225701 (2010).

[46] M. Sperl, W. T. Kranz, and A. Zippelius, Europhys. Lett. 98, 28001 (2012).

[47] W. T. Kranz, M. Sperl, and A. Zippelius, Phys. Rev. E 87, 022207 (2013).

[48] J. Talbot, R. D. Wildman, and P. Viot, Phys. Rev. Lett. 107, 138001 (2011).

[49] A. Gnoli, A. Petri, F. Dalton, G. Pontuale, G. Gradenigo, A. Sarracino, and A. Puglisi, Phys. Rev. Lett. 110, 120601 (2013).
[50] K. Kanazawa, T. G. Sano, T. Sagawa, and H. Hayakawa, Phys. Rev. Lett. 114, 090601 (2015).

[51] K. Kanazawa, T. G. Sano, T. Sagawa, and H. Hayakawa, J. Stat. Phys. 160, 1294 (2015).

[52] H. Hayakawa and M. Otsuki, Prog. Theor. Phys. 119, 381 (2008).

[53] K. Suzuki and H. Hayakawa, AIP Conf. Proc. 1542, 670 (2013).

[54] K. Suzuki and H. Hayakawa, AIP Conf. Proc. 1628, 457 (2014).

[55] O. Dauchot, G. Marty, and G. Biroli, Phys. Rev. Lett. 95, 265701 (2005).

[56] M. Pica Ciamarra and A. Coniglio, Phys. Rev. Lett. 103, 235701 (2009).

[57] V. Kumaran, J. Fluid Mech. 632, 109 (2009).

[58] D. J. Evans and G. P. Morriss, Statistical Mechanics of Nonequilibrium Liquids, (Cambridge University Press, Cambridge, England, 2008).

[59] L. D. Landau and E. M. Lifshitz, Mechanics (ButterworthHeinemann, London, 1976).

[60] See the Supplemental Material at http://link.aps.org/ supplemental/10.1103/PhysRevLett.115.098001 for details, which includes Refs. [61-69].

[61] L. E. Silbert, D. Ertaş, G. S. Grest, T. C. Halsey, D. Levine, and S. J. Plimpton, Phys. Rev. E 64, 051302 (2001).

[62] L. E. Silbert, J. W. Landry, and G. S. Grest, Phys. Fluids 15, 1 (2003).

[63] J. J. Brey, J. W. Dufty, and A. Santos, J. Stat. Phys. 87, 1051 (1997).

[64] J. W. Dufty, A. Baskaran, and J. J. Brey, Phys. Rev. E 77, 031310 (2008).

[65] A. Baskaran, J. W. Dufty, and J. J. Brey, Phys. Rev. E 77, 031311 (2008).

[66] S.-H. Chong, M. Otsuki, and H. Hayakawa, Phys. Rev. E 81, 041130 (2010).

[67] H. Hayakawa and M. Otsuki, Phys. Rev. E 88, 032117 (2013).

[68] J.-P. Hansen and I. R. McDonald, Theory of Simple Liquids (Academic Press, London, 2006).

[69] A. Donev, S. Torquato, and F. H. Stillinger, Phys. Rev. E 71, 011105 (2005).

[70] P. Résibois, J. Stat. Phys. 2, 21 (1970).

[71] S. Torquato, Phys. Rev. E 51, 3170 (1995).

[72] Although the scaling of $\sigma_{x y}$ must be $\left(\varphi-\varphi_{J}\right)^{1 / 2}$ if the contact network is unchanged for small strain $\gamma$, the contact network should be rearranged under a plane shear, because $\gamma=\dot{\gamma} t$ is not small even for small $\dot{\gamma}$ in the steady state in the limit $t \rightarrow \infty$. If $\gamma$ is not small, $\sigma_{x y}$ satisfies $y_{\phi}=1$ [4].

[73] R. Seto, R. Mari, J. F. Morris, and M. M. Denn, Phys. Rev. Lett. 111, 218301 (2013).

[74] H. Katsuragi and D. J. Durian, Nat. Phys. 3, 420 (2007).

[75] Y. Takehara, S. Fujimoto, and K. Okumura, Europhys. Lett. 92, 44003 (2010).

[76] H. Katsuragi and D. J. Durian, Phys. Rev. E 87, 052208 (2013).

[77] Y. Takehara and K. Okumura, Phys. Rev. Lett. 112, 148001 (2014).

[78] S. Takada and H. Hayakawa, arXiv:1504.04805.

[79] J. F. Brady, J. Chem. Phys. 99, 567 (1993). 